\title{
Body Mass Index, Waist-circumference and Cardiovascular Disease Risk Factors in Iranian Adults: Isfahan Healthy Heart Program
}

\author{
Noushin Mohammadifard', Masoud Nazem², Nizal Sarrafzadegan', Fatemeh Nouri', \\ Firouzeh Sajjadi ${ }^{3}$, Maryam Maghroun ${ }^{4}$, Hassan Alikhasi' \\ 'Isfahan Cardiovascular Research Center, ${ }^{2}$ Medicine Faculty School, ${ }^{3}$ Cardiac Rehabilitation Research Center, ${ }^{4}$ Hypertension \\ Research Center, Isfahan Cardiovascular Research Institute, Isfahan University of Medical Sciences, Isfahan, Iran
}

\begin{abstract}
Considering the main effect of obesity on chronic non-communicable diseases, this study was performed to assess the association between body mass index (BMI), waist-circumference (WC), cardiometabolic risk factors and to corroborate whether either or both BMI and WC are independently associated with the risk factors in a sample of Iranian adults. This cross-sectional study was performed on data from baseline survey of Isfahan Healthy Heart Program (IHHP). The study was done on 12,514 randomly-selected adults in Isfahan, Najafabad and Arak counties in 2000-2001. Ages of the subjects were recorded. Fasting blood glucose (FBG), 2-hour post-load glucose (2hpp), serum lipids, systolic and diastolic blood pressure (SBP and DBP), BMI, WC, smoking status, and total daily physical activity were determined. Increase in BMI and WC had a significant positive relation with the mean of FBG, 2hpp, SBP, DBP, serum lipids, except for HDL-C ( $\mathrm{p}<0.001$ for all). After adjustment for age, smoking, physical activity, socioeconomic status (SES), and BMI, the highest odds ratio (OR) (95\% CI) for diabetes mellitus (DM) according to WC was 3.13 (1.93-5.08) and 1.99 (1.15-3.44) in women and men respectively. Moreover, the highest ORs based on BMI with adjustment for age, smoking, physical activity, SES, and WC were for dyslipidaemia (DLP) [1.97 (1.58-2.45) in women and 2.96 (2.41-3.63) in men]. The use of $\mathrm{BMI}$ or WC alone in the models caused to enhance all ORs. When both BMI and WC were entered in the model, the ORs for all risk factors, in men, according to BMI, were more compared to WC. However, in women, ORs for DM and hypertension (HTN) in WC quartiles were more than in BMI quartiles. BMI is the better predictor of DM, HTN, and DLP in men compared to WC. Conversely, in women, WC is a superior predictor than BMI, particularly for DM and HTN. Furthermore, the measurement of both WC and BMI in Iranian adults may be a better predictor of traditional risk factors of CVDs compared to BMI or WC alone.
\end{abstract}

Key words: Body mass index; Diabetes mellitus; Dyslipidaemia; Hypertension; Obesity; Risk Factor; Waist-circumference; Iran

\section{INTRODUCTION}

Chronic non-communicable diseases (CNCDs), such as cardiovascular diseases (CVDs), cancers,

Correspondence and reprint requests:

Dr. Noushin Mohammadifard

Nutrition Department

Isfahan Cardiovascular Research Center

Isfahan Cardiovascular Research Institute

Isfahan University of Medical Sciences

PO Box 81465-1148

Isfahan

Iran

Email: mohammadifard@crc.mui.ac.ir

Fax: 0098-311-3373435 and diabetes mellitus (DM), are the major causes of death worldwide (1). Moreover, CVDs are the leading cause of death in Iran (2) while the prevalence of coronary artery disease (CAD) was $19.4 \%$ in Isfahan, a central city of Iran (3).

Body mass index (BMI) has been identified as a potential of CVD risk factor for a long time (4-7). Central adiposity in adults make them more susceptible to major metabolic problems, such as ischaemic heart diseases, myocardial infarction, hypertension (HTN), dyslipidaemia (DLP), and dibetes mellitus $(\mathrm{DM})$, and it cannot be indicated by BMI (8-10). It was assessed by measuring waist-circumference 
(WC) which is a better predictor than BMI for obesity outcomes $(11,12)$. According to the National Institute of Health guidelines, it is supposed that BMI and WC have independent effects on obesityrelated diseases (13). It is clear that combination of WC and BMI predicts health risks better than does BMI alone $(9,10)$; however, the reverse is uncertain.

According to Adult Treatment Panel III (ATP III) criteria, about $33.8 \%$ of Iranian adults (more than 10.6 million) were centrally obese. It was about 4 times in females compared to males (14).

Considering the importance of CNCDs and the main effect of obesity on these diseases, this study was performed to assess the association between BMI, WC, and major cardiometabolic risk factors and to corroborate whether either or both BMI and WC are independently associated with these risk factors in a sample of Iranian adults who participated in the baseline survey of Isfahan Healthy Heart Program (IHHP). IHHP was a long-term community-based interventional programme for health promotion through reduction of CVD risk factors, and hence, reduction of morbidity and mortalitydue to CVDs. The study was conducted in 3 central counties of Iran (15).

\section{MATERIALS AND METHODS}

\section{Sampling}

This is an analytical study done with data of the baseline survey of IHHP $(15,16)$. IHHP was undertaken in 3 counties of Isfahan, Najafabad, and Arak in the central part of Iran. According to the 2000 National Census, the population was 1,895,856, 275,084, and 668,531 in Isfahan, Najafabad, and Arak respectively $(15,16)$. Multistage random-sampling technique was employed based on sex, age, and settlement distributions in each community to select 12,600 adults aged $\geq 19$ years. Approximately 5-10\% of households within these clusters were randomly selected. One individual aged $\geq 19$ years per household was randomly selected. The selection criteria were: Iranian and mentally-competent individuals and also non-pregnant women $(15,16)$.

Single eligible subject within the household was selected randomly from one of the six age-groups: $19-<25,25-<35,35-<45,45-<55,55-64$, or 65 years and more (15).

\section{Data collection}

Eligible individuals had a 30-minute home interview by trained health professionals. The questionnaire included questions on socioeconomic and demographic characteristics, health knowledge, cardiovascular risk-related attitudes and behaviours regarding dietary practice, smoking, and physical activity (15). Medical and drug history of participants were obtained for DLP, DM, and HTN by well-trained physicians.

Trained nurses obtained blood samples from the participants by venipuncture from the left antecubital vein after 12-14 hours of fasting. They kept all blood samples frozen at $-20{ }^{\circ} \mathrm{C}$ to be assayed within 72 hours at the central laboratory of Isfahan Cardiovascular Research Center (ICRC) which meets the criteria of the National Reference Laboratory (a WHO-collaborating centre). Serum total cholesterol (TC) and triglyceride (TG) were determined by enzymatic method, using special kits (Immunodiagnostic, Germany) in an Elan 2000 auto-analyzer (Eppendorf, Germany). Also, HDL-C was measured by enzymatic method after precipitating the other lipoproteins with dextran sulphate magnesium chloride (17). LDL-C was calculated by using the Friedewald formula (18). Direct measurement of LDL-C was performed with a turbidimetric method for those with TG $\geq 400 \mathrm{mg} / \mathrm{dL}$. Blood sugar, including fasting blood glucose (FBG) and 2-hour postload plasma glucose (2hpp), were determined by glucose oxidase enzymatic method. To measure 2hpp, the blood sugar of non-diabetic participants was measured 2 hours after giving a syrup containing $75 \mathrm{~g}$ of glucose powder (19).

Physical measurements were done by trained medical staff with standardized methods. The weight was measured by a digital scale, with minimum necessary clothing, and recorded to the nearest $0.5 \mathrm{~kg}$. Height was measured in a standing position, without shoes, to the nearest $0.5 \mathrm{~cm}$, using a non-elastic stadiometer while the shoulders were in a normal state (20). BMI was calculated and recorded as weight in $\mathrm{kg}$ divided by height in metre squared (20). While the subjects were standing, WC and hip-circumference were measured by a tape at a level midway between the lower rib margin and iliac crest and at the point yielding the maximum circumference over the buttocks respectively (20). The blood pressure (BP) was measured by trained physicians based on standard criteria (21). These physicians were trained for a week to teach how to use sphygmomanometer and how to measure BP in a seated position. BP was measured two times in a seated position with a random-zero sphygmomanometer and an appropriate cutoff after a 5-minute rest. Average of the two measures was recorded for both systolic and diastolic BP (SBP and DBP). 


\section{Ethics}

Written informed consents were obtained from all participants, and the study was approved by the Ethical Committee of the ICRC (15).

\section{Definitions of risk factors and diseases}

DLP was defined as having at least one of the following situations: TC $\geq 240 \mathrm{mg} / \mathrm{dL}$ and/or TG $\geq 200$ $\mathrm{mg} / \mathrm{dL}$ and/or LDL-C $\geq 160 \mathrm{mg} / \mathrm{dL}$ and/or HDL-C $<40 \mathrm{mg} / \mathrm{dL}$ in men or HDL-C $<50 \mathrm{mg} / \mathrm{dL}$ in women (22) and/or medications for hypolipidaemia. DM was defined as $\mathrm{FBG} \geq 126 \mathrm{mg} / \mathrm{dL}$ and/or a $2 \mathrm{hpp}$ level of $\geq 200 \mathrm{mg} / \mathrm{dL}$ and/or medications for hypoglycaemia, using the WHO criteria (19). Individuals having SBP $\geq 140 \mathrm{mmHg}$ and/or DBP $\geq 90 \mathrm{mmHg}$ and/or using medications for BP were considered hypertensive patients (21).

\section{Statistical analysis}

Statistical analyses were performed using SPSS for Windows software (version 15; SPSS, Chicago, IL, USA) and STATA software (version 10). The significance level was set at $p$ value of $<0.05$. Comparison of the mean of variables with different quartiles of $\mathrm{BMI}$ and WC was done by analysis of covariance (ANCOVA), adjusting for the effect of age, smoking status (ever-smoker/non-smoker), total daily physical activity (METS per minute), and socioeconomic status (SES), including education and monthly income in each sex. The comparison of the mean value of BMI and WC in DM, HTN, and DLP patients who had either no other risk factors, one risk factor, or two risk factors were done by the analysis of variance (ANOVA). The chi-square test was used for comparing the prevalence of the risk factors in different quartiles of BMI and WC. The quantitative and qualitative basic characteristics were analyzed by $t$-test and chi-square test respectively.

Logistic regression analysis was also used for examining the independent and combined effects of $\mathrm{BMI}$ and WC on CVD risk factors. The odds ratios (ORs) in the $2^{\text {nd }}, 3^{\text {rd }}$, and $4^{\text {th }}$ quartile of BMI and WC were compared with the $1^{\text {st }}$ quartile as reference. ORs were adjusted for the above potential confounding variables. BMI and WC were entered into the regression model as continuous variables.

Preliminarily, the models with and without interaction effect were compared by likelihood ratio test (LR), Akaike Information Criterion (AIC), and Bayesian Information Criterion (BIC) tests. The results of these analyses confirmed that the combined model with BMI and WC should be reported without their interaction effect.
To test for linear trend of ORs and determine $\mathrm{p}$ for trend across quartile of $\mathrm{BMI}$ or $\mathrm{WC}$, we assigned the median BMI or WC to varying individuals as continuous variables in logistic regression for hypertensive vs non-hypertensive, diabetic vs non-diabetic hyperlipidaemic vs normolipidaemic subjects.

\section{RESULTS}

The study participants comprised 12,514 adults. We limited the current analysis to 12,416 individuals, including 6,081 men and 6,335 women because we did not have enough data for 98 individuals to be included in this analysis. The basic characteristics of the participants are presented in Table 1.

The adjusted mean values of BMI, WHR, FBG, 2hpp, TC, serum lipids, and BPs in different quartiles of WC based on sex are shown in Table 2. Increase in WC had significant positive relationship with increase in the mean of BMI, WHR, FBG, 2hpp, TC, TG, LDL-C, SBP, and DBP but with decrease in HDL-C ( $<<0.001$ for all). Comparison of DM, HTN, and HLP prevalence in WC quartiles according to sex is presented in Table 3. The DM, HTN, and DLP prevalence had a significant relationship with increase in WC ( $\mathrm{p}<0.001$ for all). Table 4 indicates that rise in BMI had a significant direct association with increase in the mean adjusted values of WC, WHR, FBG, 2hpp, TC, TG, LDL-C, SBP, and DBP and decrease in HDL-C. DM, HTN and DLP prevalence had a significant relationship with increase in BMI ( $\mathrm{p}<0.001$ for all) (Table 5). Adjusted ORs for HTN, DLP, and DM according to WC and BMI quartiles are presented in Table 6.

When both BMI and WC were entered in the model, the ORs for all risk factors, in men, according to BMI were comparable to WC. Moreover, ORs for DLP based on BMI were more than on WC in both the sexes. Conversely to men, ORs for DM and HTN in WC quartiles were more compared to BMI quartiles in women. The trends in ORs for all risk factors were gradually increased significantly by enhancing both $\mathrm{BMI}$ and WC quartiles ( $\mathrm{p}$ for trend $<0.05$ for all). The use of BMI or WC alone in the models caused to enhance all ORs (data not shown).

In total population, the mean values of WC significantly increased [ $90.6 \pm 14.4,98 \pm 12.2$, and $103.5 \pm 11.6$ (p for trend $<0.001$ )] in those with DM and no other risk factor, with one risk factor, and with two risk factors respectively. These were $93.2 \pm 13.7,98 \pm 13.3$, and 103.6 \pm 11.6 respectively in hypertensive subjects ( $\mathrm{p}$ for trend $<0.001$ ). In dyslipidaemic subjects, the mean level of WC were $90.5 \pm 12.7,98.1 \pm 13.2$, 


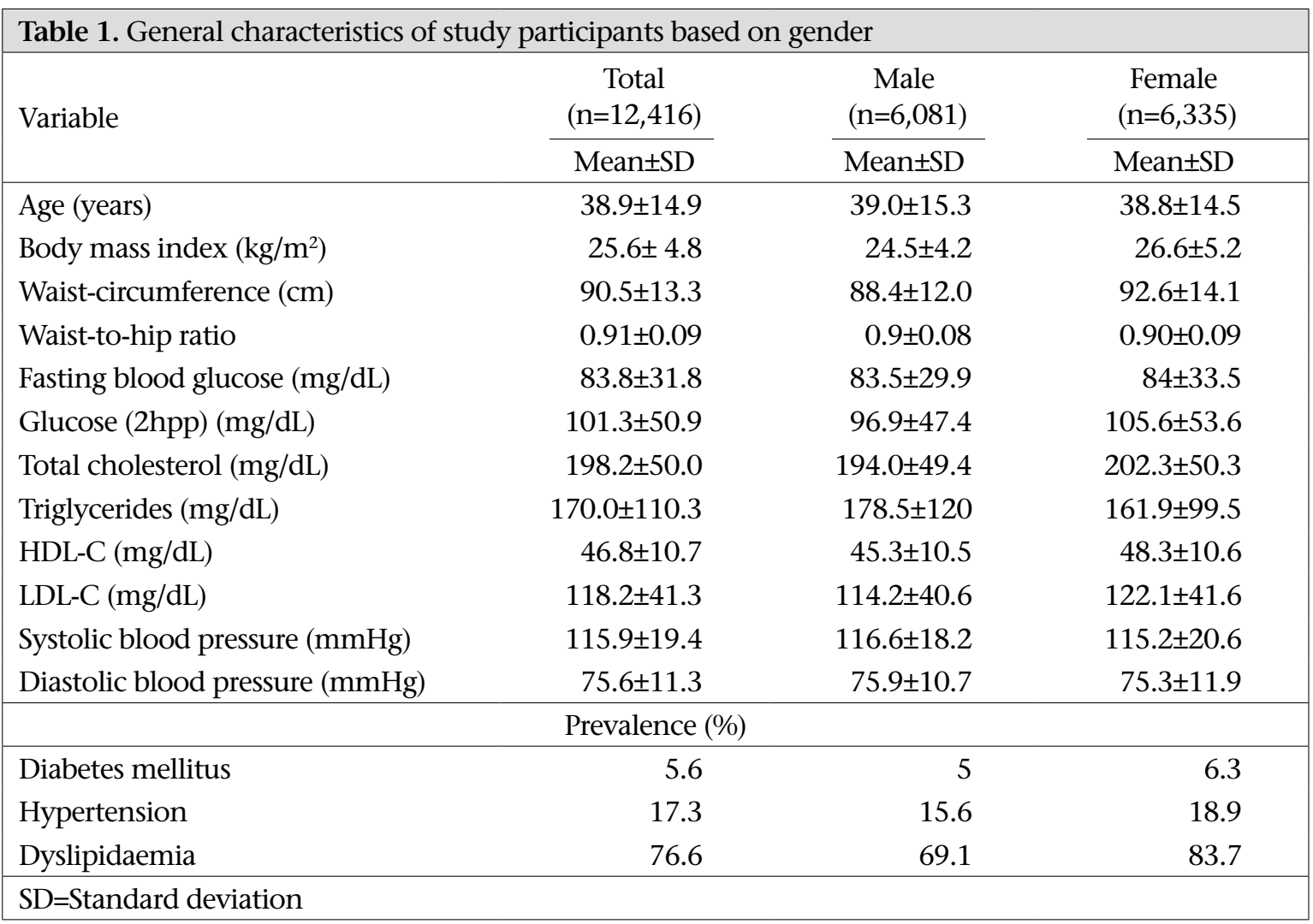

and 103.6 \pm 11.6 respectively ( $p$ for trend $<0.001$ ). $\mathrm{BMI}(\mathrm{mean} \pm \mathrm{SD})$ in diabetes and no other risk factor, with one risk factor, and with two risk factors were $24.5 \pm 4.2,27.6 \pm 4.7$, and $29.4 \pm 4.5$ respectively ( $\mathrm{p}$ for trend $<0.001)$. These were $27.8 \pm 4.5,24.9 \pm 5.1$, and $26.4 \pm 4.5$ in hypertension respectively ( $\mathrm{p}$ for trend $<0.001)$. In dyslipidaemic subjects, BMI (mean \pm SD) was $25.7 \pm 4.6,27.9 \pm 5$, and $29.4 \pm 4.5$ respectively ( $p$ for trend $<0.001$ ). Morevover, the mean value of WC and BMI in diabetic, hypertensive and dyslipidaemic subjects with different numbers of CVD risk factors based on sex are illustrated in Figure 1 and 2 . All values were significant $(\mathrm{p}<0.001)$.

\section{DISCUSSION}

The results indicated that higher BMI and WC were significantly associated with HTN, DLP, and DM. Adjusted odds ratio of CVD risk factors by age, smoking, SES, and total daily physical activity showed that the occurrence of DM, HTN, and DLP is significantly related with increase in obesity indicators. As the odds ratio in the combined models with BMI and WC were less than that in the models with BMI or WC alone, we concluded that assessing both BMI and WC may be a better predictor of CVD risk factors compared to BMI or WC alone. Ying et al. reported similar results in young and middle-aged Chinese women (23).
Our results are in conformity to similar studies in Korea, India, Australia, and Singapore. In these studies, the incidence of DM and HTN was found to increase with higher levels of BMI even in those whose BMI was within normal range (10,24-27).

A study in Japan performed by Ito and his colleagues showed that the risk of DLP, including high levels of LDL-C, and TG was significantly more in those placed in the highest one-third of WC compared to those placed in the lowest onethird of this index (28). Our study illustrated that the risk of DLP is significantly higher in the highest quartile of obesity indicators, especially BMI in both the sexes. In general, there was a positive significant relationship between the quartiles and the mean of serum lipids and the prevalence of DLP. In another study done by Tanaka and his colleagues in Japan, the same finding was reported in such a way that individuals with WC in the upper quartile had significantly higher prevalence of CVD risk factors. This study noted that at least one of the CVD risk factors increased with higher levels of WC (29).

Obesity may cause insulin resistance, leading to DM, HTN, and DLP (26-28). These results can be confirmed by significant relationship between BMI and WC, and these CVD risk factors are presented in our study. 


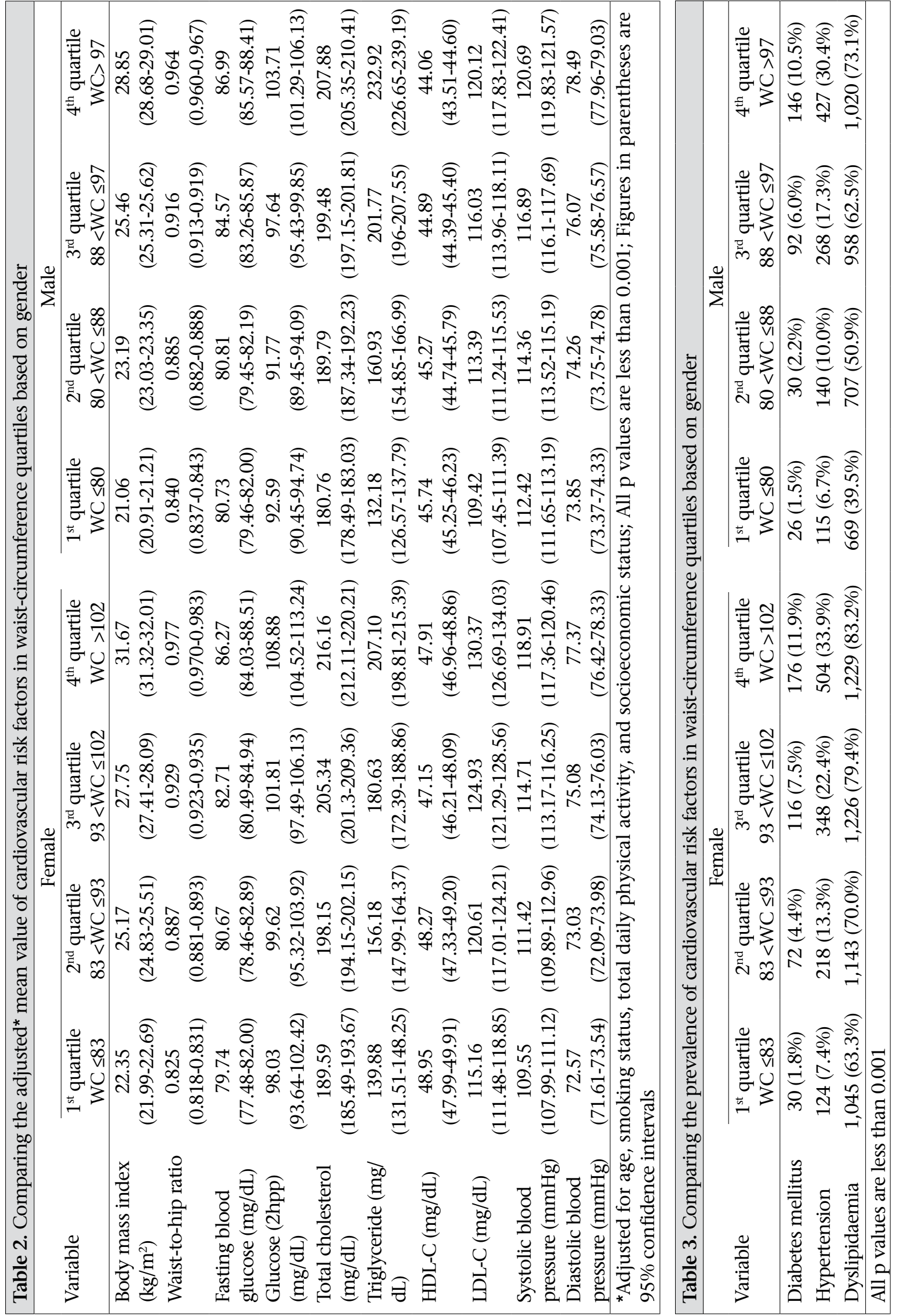




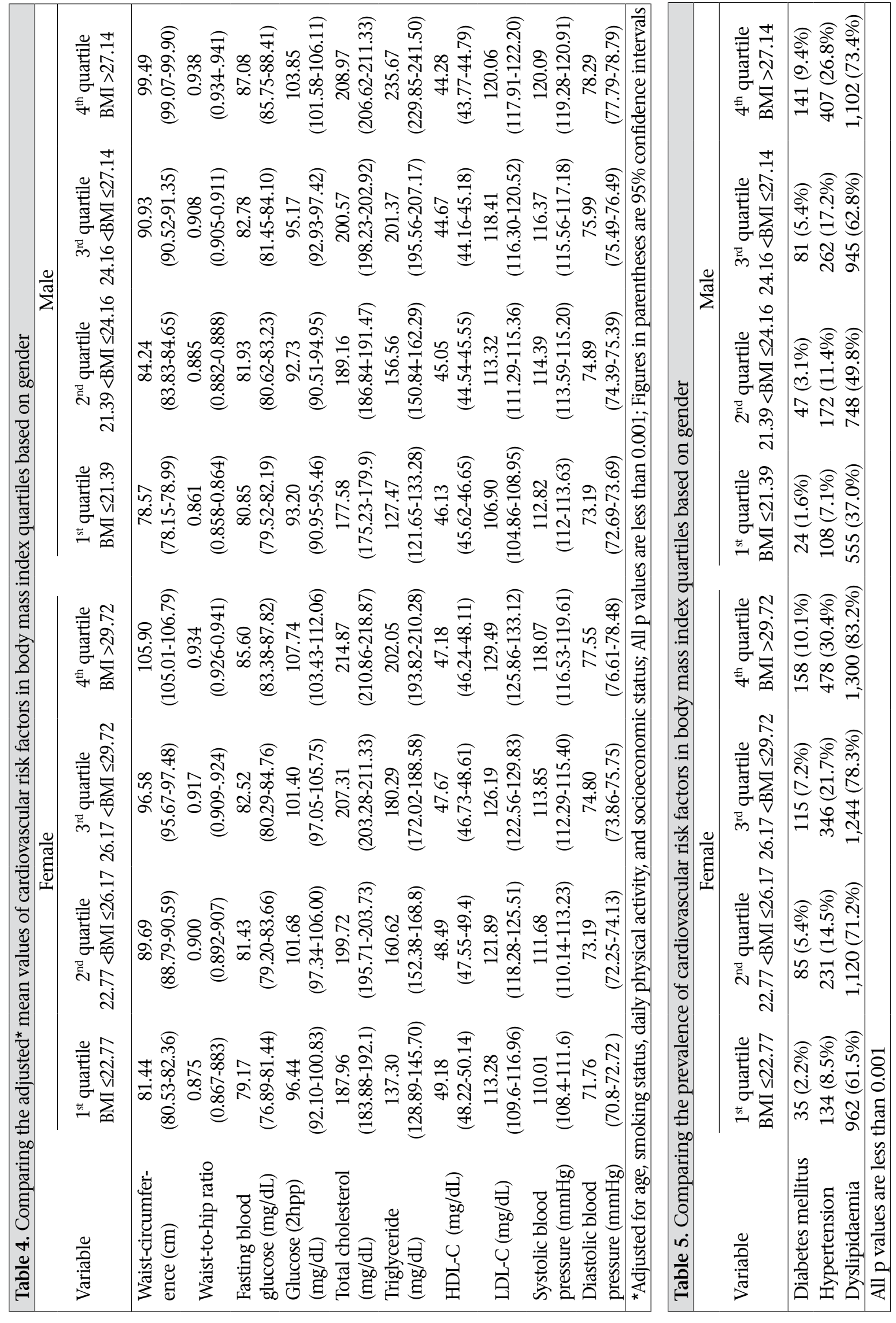




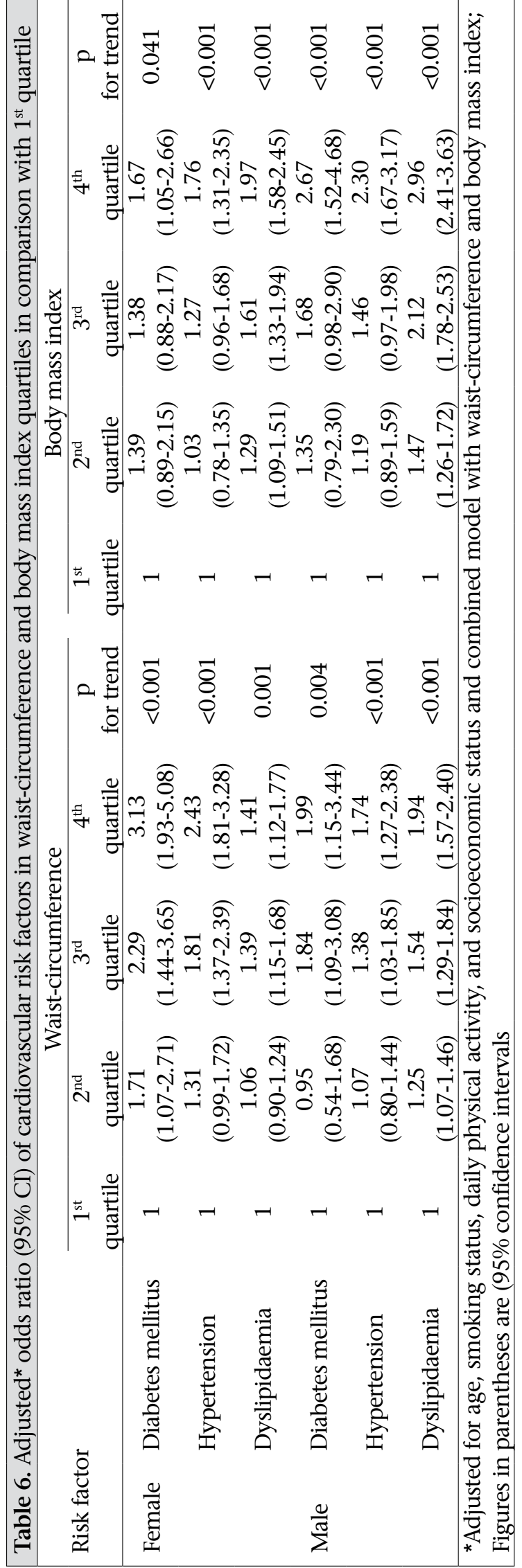

Conversely, several studies demonstrated that WC or other central obesity indicators might be superior predictors of DLP (30-32). Furthermore, according to the multiple logistic regression analysis reported herein, WC in women and BMI in men were the better predictor of DM and HTN. It may occur since the cut-points of central obesity in men based on both ATP III and International Diabetes Federation (IDF) criteria $(22,33)$ were higher than the $1^{\text {st }}$ quartile of WC, which was considered the reference value to estimate the ORs in this study. However, various studies had different results. In some of these, WC was a better and accurate measure of CVD risk (16-18). For instance, Vazquez et $a l$. reported that abdominal obesity was a stronger predictor of DM incidence than overall obesity (34). However, in the US and European Caucasians, overall obesity was a better predictor of DM (35). Also, Knowles et al. noted that WC was the best predictor of HTN in men and DM in women (36). In several studies, WC had a much stronger relationship with DM, HTN, and DLP (37-39) while in some others, both WC and BMI were worthy predictors of the CVD risk factors (11,40-41). Furthermore, a meta-analysis illustrated that BMI and WC were equally good in forecasting DM (34). Thus, measurement of both BMI and WC can improve CVD risk stratification (11).

In this study, along with the other study performed by Lee $e t$ al. on the Chinese living in Hong Kong (42), the mean BMI and WC were higher in DM, HTN and DLP subjects, with the presence of greater number of risk factors, which can demonstrate the increasing effect of both kinds of obesity indices in causing multiple risk factors.

\section{Limitations}

The use of cross-sectional data to determine the relationship between anthropometric indices and CVD risk factors is the limitation of this research work. Therefore, we were not exactly able to analyze the causal relationship between obesity and the risk factors in this study.

\section{Conclusions}

We conclude that BMI is the better predictor of DM, HTN, and DLP in men compared to WC. Conversely in women, $\mathrm{WC}$ is a superior predictor than BMI, particularly for DM and HTN subjects. So, BMI alone might be a useful indicator in Iranian men if measuring WC is difficult. Furthermore, the measurement of both WC and $\mathrm{BMI}$ in Iranian adults may be a better predictor of 
Figure 1. The mean of waist-circumference in diabetic, hypertensive and dyslipidaemic subjects with different numbers of risk factors based on sex

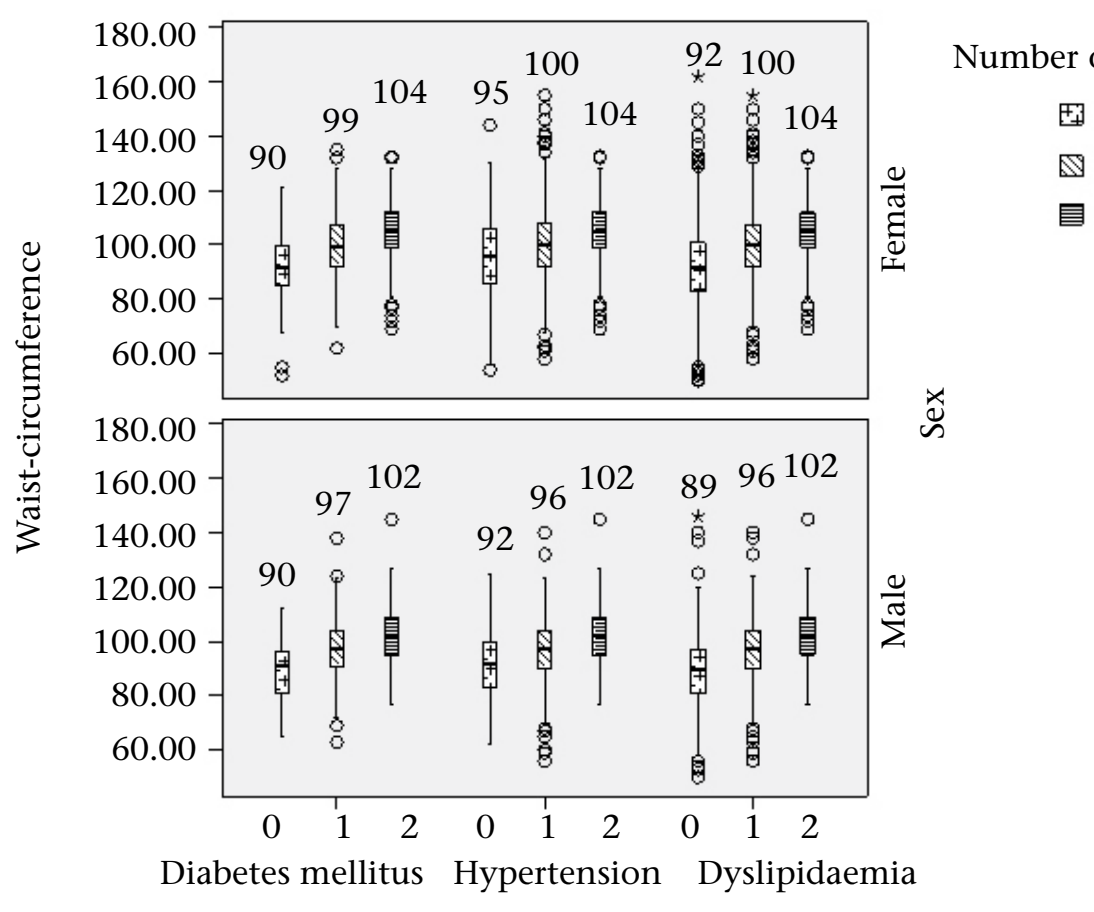

Figure 2. The mean of body mass index in diabetic, hypertensive and dyslipidaemic subjects with different numbers of risk factors based on sex

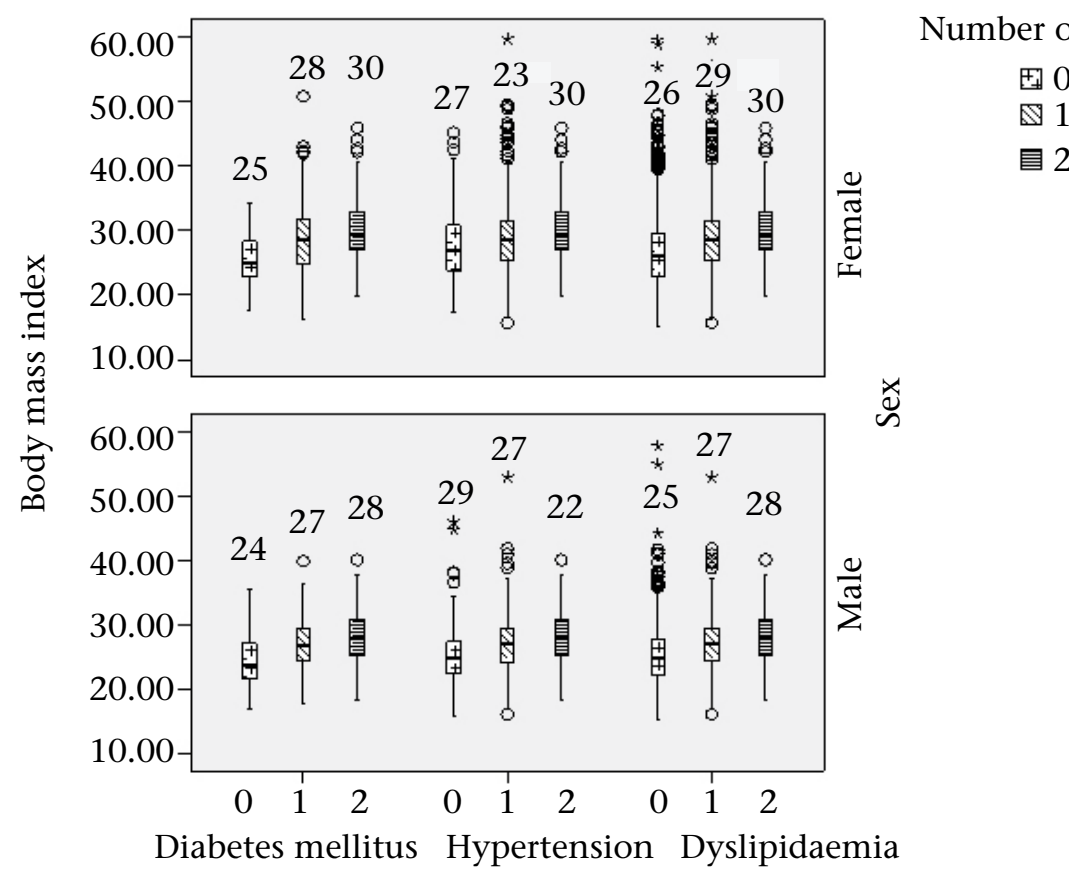

traditional risk factors of CVDs than BMI or WC alone. Therefore, it is suggested that both BMI and WC be regular measures for identification of the high-risk obese population in epidemiological studies.

\section{ACKNOWLEDGEMENTS}

This study was conducted by the ICRC (a WHOcollaborating centre) in collaboration with Isfahan Provincial Health Office, both of which are affiliat- 
ed with the Isfahan University of Medical Sciences. The work was supported by a grant (No. 31309304) from the Iranian Budget and Planning Organization as well as the Deputy for Health of the Iranian Ministry of Health and Medical Education and the Iranian Heart Foundation. We are thankful to the team of ICRC and Isfahan Provincial Health Office as well as collaborators from Najafabad Health Office and Arak University of Medical Sciences.

\section{REFERENCES}

1. World Health Organization. Prevention chronic disease: a vital investment. Geneva: World Health Organization, 2005. 182 p. (WHO global report).

2. Ghassemi H, Harrison G, Mohammad K. An accelerated nutrition transition in Iran. Public Health Nutr 2002;5:149-55.

3. Sarraf-Zadegan N, Sayed-Tabatabaei FA, Bashardoost N, Maleki A, Totonchi M, Habibi HR et al. The prevalence of coronary artery disease in an urban population in Isfahan, Iran. Acta Cardiol 1999;54:257-63.

4. Kannel WB, Cupples LA, Ramaswami R, Stokes J, 3rd, Kreger BE, Higgins M. Regional obesity and risk of cardiovascular disease; the Framingham Study. J Clin Epidemiol 1991;44:183-90.

5. Sung KC, Ryu S, Reaven GM; the Health Screening Group at Kangbuk Samsung Hospital. Relationship between obesity and several cardiovascular disease risk factors in apparently healthy Korean individuals: comparison of body mass index and waist circumference. Metabol Clin Exp 2007;56:297-303.

6. Cui R, Iso H, Toyoshima H, Date C, Yamamoto A, Kikuchi $\mathrm{S}$ et al.; JACC study group. Body mass index and mortality from cardiovascular disease among Japanese men and women: the JACC study. Stroke 2005;36:1377-82.

7. Gregg EW, Cheng YJ, Cadwell BL, Imperatore G, Williams DE, Flegal KM et al. Secular trends in cardiovascular disease risk factors according to body mass index in US adults. JAMA 2005;293:1868-74.

8. Janiszewski PM, Janssen I, Ross R. Does waist circumference predict diabetes and cardiovascular disease beyond commonly evaluated cardiometabolic risk factors? Diabetes Care 2007;30:3105-9.

9. Wang Z, Hoy WE. Waist circumference, body mass index, hip circumference and waist-to-hip ratio as predictors of cardiovascular disease in Aboriginal people. Eur J Clin Nutr 2004;58:888-93.

10. Dalton M, Cameron AJ, Zimmet PZ, Shaw JE, Jolley D, Dunstan DW et al.; AusDiab Steering Committee. Waist circumference, waist-hip ratio and body mass index and their correlation with cardiovascular disease risk factors in Australian adults. J Intern Med 2003;254:555-63.

11. Wildman RP, Gu D, Reynolds K, Duan X, Wu X, He J. Are waist circumference and body mass index independently associated with cardiovascular disease risk in Chinese adults? Am J Clin Nutr 2005;82:1195-202.

12. Goodpaster BH, Krishnaswami S, Harris TB, Katsiaras A, Kritchevsky SB, Simonsick EM et al. Obesity, regional body fat distribution, and the metabolic syndrome in older men and women. Arch Intern Med 2005;165:777-83.

13. National Institutes of Health. Clinical guidelines on the identification, evaluation, and treatment of overweight and obesity in adults-the evidence report. Obes Res 1998;6(Suppl 2):51S-209S.

14. Esteghamati A, Meysamie A, Khalilzadeh O, Rashidi A, Haghazali M, Asgari F et al. Third national surveillance of risk factors of non-communicable diseases (SuRFNCD-2007) in Iran: methods and results on prevalence of diabetes, hypertension, obesity, central obesity, and dyslipidaemia. BMC Public Health 2009;9:167.

15. Sarraf-Zadegan N, Sadri G, Afzali HM, Baghaei M, Fard NM, Shahrokhi S et al. Isfahan Healthy Heart Program: a comprehensive integrated community-based programme for cardiovascular disease prevention and control. Design, methods and initial experience. Acta Cardiol 2003;58:309-20.

16. Sarrafzadegan N, Baghaei A, Sadri G, Kelishadi $\mathrm{R}$, Malekafzali H, Boshtam M et al. Isfahan Healthy Heart Program: evaluation of comprehensive, community-based interventions for non-communicable disease prevention. Prev Control J 2006;2:73-84.

17. Warnick GR, Benderson J, Albers JJ. Dextran sulfate- $\mathrm{Mg}^{2+}$ precipitation procedure for quantitation of high-density-lipoprotein cholesterol. Clin Chem 1982;28:1379-88.

18. Friedewald WT, Levy RI, Fredrickson DS. Estimation of the concentration of low-density lipoprotein cholesterol in plasma, without use of the preparative ultracentrifuge. Clin Chem 1972;18:499-502.

19. World Health Organization. WHO draft protocol and manual of operations population survey for cardiovascular disease risk factors in the Eastern Mediterranean Region. Alexandria: World Health Organization, $1995.35 \mathrm{p}$.

20. National Institutes of Health. The practical guide: identification, evaluation and treatment of overweight and obesity in adults. Bethesda, MD: National Institutes of Health, 2000. $80 \mathrm{p}$.

21. Chobanian AV, Bakris GL, Black HR, Cushman WC, Green LA, Izzo JL, Jr. et al.; National High Blood Pres- 
sure Education Program Coordinating Committee. Seventh report of the Joint National Committee on Prevention, Detection, Evaluation, and Treatment of High Blood Pressure. Hypertension 2003;42:1206-52.

22. National Institutes of Health. Third Report of the National Cholesterol Education Program (NCEP) Expert Panel on detection, evaluation, and treatment of high blood cholesterol in adults (Adult Treatment Panel III): executive summary. Bethesda, MD: National Institutes of Health, 2001. 28 p. (NIH publication no. 01-3670).

23. Ying X, Song Z-y, Zhao C-j, Jiang Y. Body mass index, waist circumference, and cardiometabolic risk factors in young and middle-aged Chinese women. J Zhejiang Univ Sci B 2010;11:639-46.

24. Deurenberg-Yap M, Chew SK, Deurenberg P. Elevated body fat percentage and cardiovascular risks at low body mass index levels among Singaporean Chinese, Malays and Indians. Obes Rev 2002;3:209-15.

25. Snehalatha C, Viswanathan V, Ramachandran A. Cutoff values for normal anthropometric variables in Asian Indian adults. Diabetes Care 2003;26:1380-4.

26. Vikram NK, Pandey RM, Misra A, Sharma R, Devi JR, Khanna N. Non-obese (body mass index $<25 \mathrm{~kg} / \mathrm{m}^{2}$ ) Asian Indians with normal waist circumference have high cardiovascular risk. Nutrition 2003;19:503-9.

27. Park HS, Yun YS, Park JY, Kim YS, Choi JM. Obesity, abdominal obesity, and clustering of cardiovascular risk factors in South Korea. Asia Pac J Clin Nutr 2003;12:411-8.

28. Ito H, Nakasuga K, Ohshima A, Sakai Y, Maruyama T, Kaji Y et al. Excess accumulation of body fat is related to dyslipidaemia in normal-weight subjects. Int J Obes 2004;28:242-7.

29. Tanaka S, Togashi K, Rankinen T, Pérusse L, Leon AS, Rao DC et al. Is adiposity at normal body weight relevant for cardiovascular disease risk? Int J Obes 2002;26:176-83.

30. Dobbelsteyn CJ, Joffres MR, MacLean DR, Flowerdew G; The Canadian Heart Health Surveys Research Group. A comparative evaluation of waist circumference, waist-to-hip ratio and body mass index as indicators of cardiovascular risk factors. The Canadian Heart Health Surveys. Int J Obes 2001;25:652-61.

31. Pouliot MC, Després JP, Lemieux S, Moorjani S, Bouchard C, Tremblay A et al. Waist circumference and abdominal sagittal diameter: best simple anthropometric indexes of abdominal visceral adipose tissue accumulation and related cardiovascular risk in men and women. Am J Cardiol 1994;73:460-8.
32. Bertsias G, Mammas I, Linardakis M, Kafatos A. Overweight and obesity in relation to cardiovascular disease risk factors among medical students in Crete, Greece. BMC Public Health 2003;3:3.

33. International Diabetes Federation. The IDF consensus worldwide definition of the metabolic syndrome. Brussels: International Diabetes Federation, 2006. 23 p.

34. Vazquez G, Duval S, Jacobs DR, Jr., Silventoinen K. Comparison of body mass index, waist circumference, and waist/hip ratio in predicting incident diabetes: a meta-analysis. Epidemiol Rev 2007;29:11528.

35. Chan JM, Rimm EB, Colditz GA, Stampfer MJ, Willett WC. Obesity, fat distribution, and weight gain as risk factors for clinical diabetes in men. Diabetes Care 1994;17:961-9.

36. Knowles KM, Paiva LL, Sanchez SE, Revilla L, Lopez $\mathrm{T}$, Yasuda MB et al. Waist circumference, body mass index, and other measures of adiposity in predicting cardiovascular disease risk factors among Peruvian adults. Int J Hypertens 2011;2011:931402.

37. Foucan L, Hanley J, Deloumeaux J, Suissa S. Body mass index (BMI) and waist circumference (WC) as screening tools for cardiovascular risk factors in Guadeloupean women. J Clin Epidemiol 2002;55:990-6.

38. Canoy D, Boekholdt SM, Wareham N, Luben R, Welch A, Bingham $S$ et al. Body fat distribution and risk of coronary heart disease in men and women in the European Prospective Investigation into Cancer and Nutrition in Norfolk cohort: a population-based prospective study. Circulation 2007;116:2933-43.

39. Chen L, Peeters A, Magliano DJ, Shaw JE, Welborn TA, Wolfe R et al. Anthropometric measures and absolute cardiovascular risk estimates in the Australian Diabetes, Obesity and Lifestyle (AusDiab) Study. Eur J Cardiovasc Prev Rehabil 2007;14:740-5.

40. Rezende FAC, Rosado LEFPL, de Cássia Lanes Ribeiro $\mathrm{R}$, de Carvalho Vidigal F, Vasques ACJ, Bonard IS et al. Body mass index and waist circumference: association with cardiovascular risk factors. Arq Bras Cardiol 2006;87:728-34.

41. Paniagua L, Lohsoonthorn V, Lertmaharit S, Jiamjarasrangsi W, Williams MA. Comparison of waist circumference, body mass index, percent body fat and other measure of adiposity in identifying cardiovascular disease risks among Thai adults. Obes Res Clin Pract 2008;2:215-23.

42. Lee ZSK, Critchley JAJH, Ko GTC, Anderson PJ, Thomas GN, Young RP et al. Obesity and cardiovascular risk factors in Hong Kong Chinese. Obe Rev 2002;3:173-82. 